\title{
Mortality in virulent canine babesiosis is associated with a consumptive coagulopathy
}

Amelia Goddard ${ }^{\mathrm{a},{ }^{*},}$, Bo Wiinberg ${ }^{\mathrm{b}}$, Johan P. Schoeman a , Annemarie T. Kristensen ${ }^{\mathrm{b}}$, Mads Kjelgaard-Hansen ${ }^{\mathrm{b}}$

${ }^{a}$ Department of Companion Animal Clinical Studies, Faculty of Veterinary Science, University of Pretoria, South Africa

${ }^{\mathrm{b}}$ Department of Veterinary Clinical and Animal Sciences, Faculty of Life Sciences, University of Copenhagen, Denmark

* Corresponding author. Tel.: +27 125298293.

E-mail address: amelia.goddard@up.ac.za (A. Goddard)

\begin{abstract}
The inflammatory response to infection can activate the coagulation system via complex interactions. If uncontrolled, this may lead to a consumptive coagulopathy, which has been identified as a major risk factor for poor outcome in both human and canine medicine. This study was undertaken to prospectively determine whether the presence of a consumptive coagulopathy in dogs with Babesia rossi infection is related to mortality. A prospective, cross-sectional, observational study was performed. Seventy-two client-owned dogs diagnosed with canine babesiosis were included. Diagnosis was confirmed by polymerase chain reaction and reverse line blot and dogs infected with Babesia vogeli or Ehrlichia canis were excluded. Blood samples were collected at admission. Coagulation factor-, antithrombin (AT)-, and protein C (PC) activity, prothrombin time (PT), activated partial thromboplastin time (aPTT), fibrinogen and Ddimer concentrations were measured. The mortality rate was $18 \%(13 / 72)$ and results between non-survivors and survivors were compared. The median activities of all the coagulation factors were significantly lower in the non-survivors compared to the survivors. The median PT and aPTT were significantly longer in the non-survivors compared to the survivors. The median AT activity was not significantly different; however, the median PC activity was significantly
\end{abstract}


decreased in the non-survivors. The median D-dimer concentration was significantly higher in the non-survivors compared to the survivors. This study showed that dogs that died from B.canis infection suffered from a more severe consumptive coagulopathy compared to survivors, characterized by procoagulant activation, inhibitor consumption, and increased fibrinolytic activity.

Keywords: Babesia rossi, Coagulopathy, DIC, Prognosis

\section{Introduction}

Babesia rossi is a virulent protozoan, capable of infecting the dog and babesiosis caused by this organism is a severe and highly prevalent disease in South Africa. The mortality rate in complicated cases is around $10 \%$, of which $80 \%$ die within the first 24 hours (Keller et al., 2004;

Nel et al., 2004; Schoeman et al., 2007). Clinical signs of canine babesiosis vary and include peracute, acute, chronic, subclinical and atypical presentations (Schoeman, 2009). Acute babesiosis is the most common presentation in South Africa and it typically manifests as fever, lethargy, anemia, thrombocytopenia and splenomegaly, with or without hemoglobinuria (Schoeman, 2009). The most common complications of acute babesiosis include hemoconcentration, disseminated intravascular coagulation (DIC), icterus and hepatopathy, secondary immune-mediated hemolytic anemia (IMHA), acute kidney injury (AKI), pancreatitis and pulmonary edema (Jacobson and Clark, 1994; Jacobson, 2006).

It is clear from the literature that the disease caused by Babesia spp. is a severe, often lethal, blood-borne multisystemic disease caused by an exuberant and ineffective immune response that may result in death through complete organ failure (Ahmed, 2002; Clark and Jacobson, 1998; Hemmer et al., 2000; Welzl et al., 2001; Wright et al., 1989). C-reactive protein (CRP), a marker 
of the inflammatory response, has been identified as a significant predictor of outcome, in conjunction with hypoglycemia (Köster et al., 2009). The inflammatory response to infection can activate the coagulation system via complex interactions and result in a consumptive coagulopathy (Esmon et al., 1999; Laforcade et al., 2003; Weiss and Rashid, 1998). Coagulation derangement, specifically hypercoagulability, is considered likely in a number of systemic diseases affecting small animals (Donahue and Otto, 2005; Kristensen et al., 2008; Otto et al., 2000; Wiinberg et al., 2008; Wiinberg et al., 2009). If uncontrolled, the hypercoagulable state may lead to DIC, which has been identified as a major risk factor for poor outcome in both human and canine medicine (Laforcade et al., 2003; Weiss and Rashid, 1998). The criteria for the definition of DIC in people, established by the International Society of Thrombosis and Hemostasis (ISTH), include procoagulant activation, inhibitor consumption, and increased fibrinolytic activity (Bick et al., 1999; Wiinberg et al., 2008). Clinical signs associated with DIC vary considerably and can range from no signs (non-overt DIC) to signs of organ failure, secondary to microvascular thrombosis, and overt hemorrhage (overt DIC) (Bick et al., 1999; Wiinberg et al., 2008). A recent study in dogs has indicated that the same criteria can be used for diagnosing DIC in dogs and a significant difference in plasma based assays was observed between survivors and non-survivors (Wiinberg et al., 2010).

Dogs naturally infected with $B$. rossi suffer from Systemic Inflammatory Response Syndrome (SIRS) (Welzl et al., 2001) and DIC has been described as a complication that is likely involved in the severe multi organ damage observed (Moore and Williams, 1979), but whether DIC and the severity of the associated consumptive coagulopathy is a predictor of outcome is unknown. In this study we hypothesized that mortality in dogs infected with B. rossi 
is associated with the presence and severity of consumptive coagulopathy, characterized by procoagulant activation, inhibitor consumption, and increased fibrinolytic activity.

\section{Materials and methods}

This prospective, cross-sectional, observational study included client-owned dogs diagnosed with canine babesiosis that were sick enough to be admitted to the ICU. The research protocol was approved by the University of Pretoria's Animal Use and Care Committee. Owner consent was obtained for enrolment of all the cases in this study. Infection with B. rossi was confirmed by demonstration of the intra-erythrocytic trophozoite on a stained thin bloodsmear as well as by polymerase chain reaction (PCR) and reverse line blot (RLB). Dogs infected with B. vogeli or Ehrlichia canis, and dogs euthanized for reasons other than poor prognosis were not included.

\section{Animals}

All cases with a presenting packed cell volume $(\mathrm{PCV})<15 \%$, complicated or uncomplicated, were hospitalized. To satisfy the criteria of a complicated case, dogs had to suffer from one or more of the following complications: AKI (oliguria/anuria and persistently elevated serum creatinine concentration despite appropriate fluid therapy); cerebral babesiosis (neurological signs that can not be attributed to any other cause); icterus indicating hepatopathy with cholestasis (icterus, bilirubinuria, bilirubinemia, raised ALT and ALP); secondary IMHA (icterus, warm in-saline agglutination and/or marked spherocytosis); acute respiratory distress (dyspnea, adventitious lung sounds, radiological evidence of lung consolidation or edema, and blood-gas evidence of ventilation-perfusion mismatch); hemoconcentration (normal or raised PCV in association with intravascular hemolysis) and pancreatitis (icterus, vomiting, diarrhea, 
melena, elevated serum amylase and lipase concentrations, ultrasonographic evidence of acute pancreatitis) (Jacobson, 2006). Dogs received standard care for canine babesiosis, which included antibabesial treatment with diminazene (Berenil RTU $0.07 \mathrm{~g} / \mathrm{mL}$, Intervet), and blood transfusions with packed red cells (PRC) as needed. In addition, any complications were treated at the discretion of the attending clinician. Outcome was recorded as short-term survival (i.e. until discharge), or death/euthanasia.

\section{Sample collection}

At admission, prior to any treatment, blood samples were collected from the jugular vein from each dog with a 21-gauge needle by careful venipuncture with minimum stasis. Blood samples were collected into serum, citrated and EDTA vacutainer plastic tubes (BD Vacutainer tube, The Scientific Group). The blood samples were collected in the order described above. The $3 \mathrm{~mL}$ sodium citrate tube was inverted carefully after sampling to ensure mixing of the $3.2 \%$ trisodium citrate and blood in a 1:9 ratio. The EDTA sample was used for complete blood count (CBC). The sodium citrate sample was used to perform the plasma coagulation profile. The citrate sample was centrifuged at $2100 \mathrm{~g}$ for 8 minutes, after which the plasma was aliquoted and stored at $-80^{\circ} \mathrm{C}$.

\section{Coagulation profile}

The plasma coagulation profile analysis was performed as a batch and included plasma coagulation factor activity (i.e. factors II, V, VII, VIII, IX, X, XI, XII and fibrinogen), prothrombin time (PT), activated partial thromboplastin time (aPTT); inhibitors (antithrombin (AT) activity, protein $\mathrm{C}$ (PC) activity); and fibrinolysis activation (D-dimer concentration). A 
CBC was performed on an automated cell counter (ADVIA 2120, Siemens). For the coagulation factor activities, PT, aPTT, AT- and PC activity, fibrinogen and D-dimer concentrations the protocols suggested by the manufacturers were followed. The plasma coagulation factor activities, PT, aPTT, AT- and PC activity and fibrinogen concentration were assessed using an automated coagulometric analyzer (ACL top 500, Instrumentation Laboratory). Plasma D-dimer concentration was measured using an immunometric flow-through principle (Nycocard Reader II, ILEX) (Wiinberg et al., 2007). The stored citrated plasma samples were transported to the Veterinary Clinical Pathology laboratory, University of Copenhagen, Denmark on dry ice and transit time for the shipment was $<24$ hours. All assays on the plasma were performed 21 months after the start of collection. All assays were calibrated according to the manufacturers' recommendations for human purposes. All coagulation factor-, AT- and PC activities were recorded as species-specific activities as assays were calibrated with canine material. Diagnosis of a consumptive coagulopathy was based on the criteria for the approach to DIC in people defined by ISTH, which has previously been validated in dogs; i.e. indications of pro-coagulant activation (prolonged PT, prolonged aPTT, decreased coagulation factor activity, low platelet count), inhibitor consumption (low AT, low PC) and increased fibrinolytic activity (high DDimer) (Bick et al., 1999; Taylor FB Jr. et al, 2001; Wiinberg et al., 2010). For this study, overt DIC was defined as the presence of hemostatic abnormalities together with overt hemorrhage. Non-overt DIC was defined as the presence of hemostatic abnormalities in the absence of gross clinical signs of hemorrhage (Bick et al., 1999; Wiinberg et al., 2008). 
DNA extraction and PCR

DNA was extracted from $200 \mu \mathrm{L}$ of each whole blood sample using a blood and tissue extraction kit (QIAmp blood and tissue extraction kit, Qiagen) according to the manufacturer's instructions. Molecular diagnosis of B. rossi and exclusion of other Babesia species, Ehrlichia and Anaplasma species was performed using PCR and RLB (Matjila et al., 2004). PCR was conducted with a set of primers that amplified a $460-540$ base pair fragment of the $18 \mathrm{~S} \mathrm{SSU}$ rRNA spanning the V4 region, a region conserved for Babesia and Theileria. The Ehrlichia PCR amplified the V1 hypervariable region of the 16S SSU rRNA (Bekker et al., 2002; Schouls et al., 1999). The membrane used for RLB included probes for B. vogeli, B. rossi, B. canis and E. canis.

\section{Statistical analysis}

Statistical analysis was performed using a commercial software package (SPSS Statistics 17.0 software, SPSS Inc.). The data was checked for normal distribution using the Shapiro Wilk's test. Medians of the non-survivors and survivors were compared using the Mann-Whitney U test and gender proportions between groups were compared using the Chi square test. Level of significance was set to $P<0.05$. Data is presented as percentages or median and interquartile range (IQR).

\section{Results}

A total of 75 dogs were admitted over the study period of which only 72 were included in the study. The mortality fraction was $18 \%(13 / 72)$. Of the 13 non-survivors, one dog was diagnosed with cerebral babesiosis and anuria and was euthanized due to a poor prognosis. The 
complications associated with the remaining 12 non-survivors included: acute respiratory distress together with hypoglycemia (3); acute respiratory distress (1); cerebral babesiosis (2); hypoglycemia (2); icterus (3); icterus with melena and hypoglycemia (1). Of the 72 dogs, 40 $(56 \%)$ were male and $32(44 \%)$ were female with no significant difference in gender distribution between the two groups $(P=0.27)$. The median patient age was 12 months $(7-48)$. The median age of the non-survivors was 24 months (5-96) and of the survivors 12 months (7-48). The median age between the two groups did not differ significantly $(P=0.68)$.

Table 1: Median and interquartile range of several hemostatic analytes in 59 surviving and 13 non-surviving dogs infected with Babesia rossi.

\begin{tabular}{|l|l|l|l|l|}
\hline Parameter & $\begin{array}{l}\text { Reference } \\
\text { intervals }\end{array}$ & Non-survivors & Survivors & P-value \\
\hline Procoagulant activation & $300-500 \times 10^{9} / \mathrm{L}$ & $3.9(0.2-36.3)$ & $7.5(2.9-48.8)$ & 0.21 \\
\hline Platelets & $80-150 \%$ & $40.5(30.9-70.9)$ & $76.7(61.7-87.5)$ & $0.003^{*}$ \\
\hline Factor II & $80-140 \%$ & $56.8(29.9-88.1)$ & $106.4(85.0-140.0)$ & $<0.001^{*}$ \\
\hline Factor V & $80-150 \%$ & $46.9(22.5-77.0)$ & $73.4(52.7-103.5)$ & $0.015^{*}$ \\
\hline Factor VII & $80-150 \%$ & $60.3(44.4-94.7)$ & $109.7(85.7-144.2)$ & $<0.001^{*}$ \\
\hline Factor VIII & $80-150 \%$ & $56.5(38.8-87.5)$ & $101.7(76.6-118.4)$ & $<0.001^{*}$ \\
\hline Factor IX & $80-120 \%$ & $35.4(20.2-58.8)$ & $73.9(51.9-93.1)$ & $<0.001^{*}$ \\
\hline Factor X & $80-150 \%$ & $43.4(33.0-65.2)$ & $74.9(58.0-90.4)$ & $0.002^{*}$ \\
\hline Factor XI & $80-150 \%$ & $48.9(24.3-69.9)$ & $77.4(60.5-93.9)$ & $0.002^{*}$ \\
\hline Factor XII & $6.0-9.5 \mathrm{sec}$ & $10.2(8.3-12.6)$ & $8.0(7.4-8.9)$ & $0.002^{*}$ \\
\hline PT & $9-12.5 \mathrm{sec}$ & $20.5(15.1-35.5)$ & $15.5(13.8-18.5)$ & $0.016^{*}$ \\
\hline aPTT & $1-4 \mathrm{~g} / \mathrm{L}$ & $3.7(2.4-6.5)$ & 0.25 \\
\hline Fibrinogen & \multicolumn{5}{|l}{} & \\
\hline Inhibitor consumption & $80-150 \%$ & $73.5(48.5-103.3)$ & $97.0(82.0-113.0)$ & 0.07 \\
\hline Antithrombin & $75-150 \%$ & $44.0(26.5-75.5)$ & $74.0(56.0-105.0)$ & $0.029^{*}$ \\
\hline Protein C & $0-0.5 \mathrm{mg} / \mathrm{L}$ & $1.6(1.3-2.5)$ & $0.4(0.2-0.8)$ & \\
\hline Increased fibrinolytic activity & & \\
\hline D-Dimer & \multicolumn{5}{|l|}{} & \\
\hline
\end{tabular}

*Denote statistically significant differences between groups All results given in percent represent the percent activity relative to a normal pool of canine plasma. 
Table 1 contains a summary of all the data, including the median and the interquartile range for each parameter. There were significant differences between the non-survivors and the survivors, with the non-survivors presenting with a significantly more severe consumptive coagulopathy. The median activities of all the individual coagulation factors were significantly lower in the non-survivors compared to the survivors. The median PT and aPTT were significantly longer in the non-survivors compared to the survivors. Median fibrinogen concentration was not significantly different between the two groups. The median AT activity was not significantly different between the two groups; however, the median PC activity was significantly lower in the non-survivors compared to the survivors. The median D-dimer concentration was significantly higher in the non-survivors than in the dogs that survived. The median platelet count was low in all infected animals, but not significantly different between the non-survivors and the survivors. No significant platelet aggregation, which could have contributed to the low platelet count, was reported in any of the samples.

\section{Discussion}

This study demonstrated the presence of a more severe consumptive coagulopathy characterized by significantly increased procoagulant activation, inhibitor consumption and fibrinolytic activity in dogs infected with $B$. rossi that did not survive, without gross clinical evidence of hemorrhage. These findings support the presence of non-overt DIC in these dogs according to previously reported criteria for dogs (Wiinberg et al., 2010). The mortality rate was higher in this study compared to previous reports; however, this study only included dogs that were sick enough to be admitted. When only admitted cases are compared, the mortality rate is similar to that reported in a previous study (Schoeman et al., 2007). 
The coagulation factor activities in the dogs that died, specifically factor II (thrombin), were significantly lower compared to those that survived, likely resulting from inflammatory induced coagulation activation and consumption caused by the infection. Inflammatory cytokines initiate coagulation events at sites of inflammation through various mechanisms which include expression of tissue factor (TF), altered thrombogenicity of endothelial surfaces, and activation of platelets (Weiss and Rashid, 1998). TF is the primary physiologic initiator of coagulation and is not normally expressed by cells that are in direct contact with blood. However endothelial cells, neutrophils and monocytes express TF on their surfaces in response to a variety of stimuli, including endotoxin, interleukin-6 (IL-6), tumor necrosis factor (TNF), IL-1 and immune complexes (Brady and Otto, 2001; DelGiudice and White, 2009; Hoffman and Monroe, 2001; Weiss and Rashid, 1998). TF thus plays an important role in the initiation of inflammationinduced coagulation (Levi et al., 2006). Thrombin plays a central role in propagation of coagulation, anticoagulation and fibrinolysis. The most important role of thrombin in coagulation is binding soluble fibrinogen to form insoluble fibrin monomers which then polymerize spontaneously. The presence of thrombin also has a positive feedback effect on the formation of activated factors Va, VIIa, VIIIa and XIa, which propagates thrombin formation. The more significant decrease in coagulation factor activities in the non-survivors was mirrored in the significantly prolonged PT and aPTT for this group which are specific assays for plasma coagulation protease activity. This finding is most likely due to the marked systemic inflammatory response reported in babesiosis (Ahmed, 2002; Clark and Jacobson, 1998; Hemmer et al., 2000; Welzl et al., 2001; Wright et al., 1989).

There was no significant difference in median AT activity between the two groups; however, the median PC activity was significantly lower in the non-survivors compared to the survivors, 
indicating increased inhibitor consumption or inactivation in the dogs that died.

Thrombomodulin is expressed at high levels on endothelial cells, especially in the capillaries (Hoffman and Monroe, 2001). Thrombin that escapes into the circulation from a site of injury is either inhibited by circulating AT or binds to thrombomodulin on intact vascular endothelium, upon which the specificity of thrombin is changed, and becomes more effective at activating PC rather than clotting fibrinogen or activating platelets (Hoffman and Monroe, 2001; Weiss and Rashid, 1998). PC is localized to vascular endothelial cells and forms a complex with protein S, its co-factor, once activated by the thrombin/thrombomodulin complex. The activated protein $\mathrm{C} /$ protein S complex cleaves and inactivates endothelial surface-bound activated factors Va and VIIIa (Hoffman and Monroe, 2001; Weiss and Rashid, 1998). During inflammation endothelial function is also altered in that thrombomodulin expression is down-regulated by the interaction with inflammatory mediators. This results in decreased inactivation of thrombin and decreased activation of PC, which may lead to a procoagulant state (Hoffman and Monroe, 2001; Weiss and Rashid, 1998).

In late overt stages of DIC, fibrinogen concentrations can decrease due to depletion through unregulated cleavage by thrombin and plasmin, however the median fibrinogen level for all the dogs in this study was increased and there was no significant difference between the two groups. This increase is likely due to fibrinogen's role as a positive acute phase reactant whereby its hepatic synthesis is upregulated with systemic inflammation (Murata et al., 2004). In the nonovert phase of DIC, especially in patients with SIRS, the rate of fibrinogen synthesis can exceed fibrinogen consumption, and hyperfibrinogenemia may occur (Cerón et al., 2005; Murata et al., 2004; Scott-Moncrieff et al., 2001). The binding of thrombin to thrombomodulin competitively 
inhibits the binding of fibrinogen to thrombin, which may also have contributed to the increased fibrinogen concentration in these cases (Weiss and Rashid, 1998).

This study documented increased fibrinolysis activation in non-survivors as plasma D-dimer concentration was significantly higher in the non-survivors compared to the survivors. Fibrinolysis is an essential component of hemostasis and is initiated concurrently with coagulation (Stokol, 2003). Fibrinolysis is mediated by plasmin, which is converted from plasminogen to its active state by a variety of plasminogen activators. Insoluble cross-linked fibrin is a substrate for plasmin and produces D-dimers as a degradation product, which has been shown to be very specific for fibrinolysis (Stokol, 2003). D-dimers are formed during clot formation and are exposed when the clot is lysed by plasmin. Thus, increased D-dimer concentration represents activation of both thrombin and plasmin and is a specific marker of fibrinolysis (Smith, 2010; Stokol, 2003).

The platelet count was not significantly different between the non-survivors and the survivors in this study, likely due to the very low platelet counts in both groups, thereby masking any increased consumption on the dogs with DIC. Previous studies on $B$. canis have shown that marked thrombocytopenia was the most common hemostatic change, however very few cases show any gross clinical signs of primary hemostatic abnormalities (Rafaj et al., 2005; Rafaj et al., 2009; Ruiz et al., 2007; Schetters et al., 2009). A similar severe thrombocytopenia is seen in B. ross $i$ with platelet counts reported as low as $14 \times 10^{9} / \mathrm{L}$ being a common finding without any associated clinical signs of hemorrhage (Kettner et al., 2003). As with canine babesiosis, thrombocytopenia develops early in falciparum malaria in people but the underlying mechanisms remain unresolved (de Mast et al., 2010). 
Limitations to this study included that the investigators had no control over when in the disease process the cases were presented to the hospital, and that the study only included assays performed on citrated plasma, excluding the vital role that blood cells and endothelium play during coagulation. Therefore, a more holistic approach, such as the use of thromboelastography, should be considered to investigate the consumptive coagulopathy in canine babesiosis in the future. Other limitations include the fact that the treatment protocols (i.e. blood transfusion or not) and type of complication were not included in the statistical anlysis. However, the standard blood product used for babesiosis is PRC and most of the non-survivors (9/13) died on the day of presentation, making it unlikely that the transfusion would have made a difference to the outcome.

\section{Conclusions}

In conclusion, this study has showed that mortality in dogs infected with $B$. rossi was associated with a more severe consumptive coagulopathy characterized by procoagulant activation, inhibitor consumption and fibrinolysis activation without the presence of gross hemorrhage, thus fulfilling the requirements of a diagnosis of non-overt DIC. This suggests that the presence of significant consumptive coagulopathy is related to short-term survival.

\section{Conflict of interest statement}

None of the authors of this paper has a financial or personal relationship with other people or organisations that could inappropriately influence or bias the content of the paper. 


\section{Acknowledgements}

The authors wish to thank Dr Lisa Köster for assisting in the sample collection whilst collecting data for her own research, and all participating laboratory staff for their assistance in the study. The study was financially supported by the research fund of the Department of Companion Animal Clinical Studies, University of Pretoria. The work was presented as a scientific abstract at the Annual Forum of the American College of Veterinary Internal Medicine 2010, Anaheim, California, USA.

\section{References}

Ahmed, J.S., 2002. The role of cytokines in immunity and immunopathogenesis of pirolasmoses. Parasitology research 88, S48-50.

Bekker, C.P.J., de Vos, S., Taoufik, A., Sparagano, O.A.E., Jongejan, F., 2002. Simultaneous detection of Anaplasma and Ehrlichia species in ruminants and detection of Ehrlichia ruminantium in Amblyomma variegatum ticks by reverse line blot hybridization. Veterinary microbiology 89, 223-238.

Bick, R.L., Arun, B., Frenkel, E.P., 1999. Disseminated intravascular coagulation. Clinical and pathophysiological mechanisms and manifestations. Haemostasis 29, 111-134.

Brady, C.A., Otto, C.M., 2001. Systemic inflammatory response syndrome, sepsis, and multiple organ dysfunction. Veterinary Clinics of North America, Small Animal Practice 31, 1147-1162.

Cerón, J.J., Eckersall, P.D., Martínez-Subiela, S., 2005. Acute phase proteins in dogs and cats: current knowledge and future perspectives. Veterinary Clinical Pathology 34, 85-99.

Clark, I.A., Jacobson, L.S., 1998. Do babesiosis and malaria share a common disease process? Annals of Tropical Medicine \& Parasitology 92, 483-488.

de Mast, Q., de Groot, P.G., van Heerde, W.L., Roestenberg, M., van Velzen, J.F., Verbruggen, B., Roest, M., McCall, M., Nieman, A.E., Westendorp, J., Syafruddin, D., Fijnheer, R., van Dongen-Lases, E.C., Sauerwein, R.W., van der Ven, A.J., 2010. Thrombocytopenia in early malaria is associated with GP1b shedding in absence of systemic platelet activation and consumptive coagulopathy. British journal of haematology 151, 495-503. 
DelGiudice, L.A., White, G.A., 2009. The role of tissue factor and tissue factor pathway inhibitor in health and disease states. Journal of Veterinary Emergency and Critical Care 19, 2329.

Donahue, S.M., Otto, C.M., 2005. Thromboelastography: a tool for measuring hypercoagulability, hypocoagulability, and fibrinolysis. Journal of Veterinary Emergency and Critical Care 15, 9-16.

Esmon, C.T., Fukudome, K., Mather, T., Bode, W., Regan, L.M., Stearns-Kurosawa, D.J., Kurosawa, S., 1999. Inflammation, sepsis, and coagulation. Haematologica 84, 254-259.

Hemmer, R.M., Ferrick, D.A., Conrad, P.A., 2000. Role of T cells and cytokines in fatal and resolving experimental babesiosis: protection in TNFRp55-/- mice infected with the human Babesia WA1 parasite. Journal of Parasitology 86, 736-742.

Hoffman, M., Monroe, D.M.,3rd, 2001. A cell-based model of hemostasis. Thrombosis \& Haemostasis 85, 958-965.

Jacobson, L.S., 2006. The South African form of severe and complicated canine babesiosis: clinical advances 1994-2004. Veterinary parasitology 138, 126-139.

Jacobson, L.S., Clark, I.A., 1994. The pathophysiology of canine babesiosis: new approaches to an old puzzle. Journal of the South African Veterinary Association 65, 134-145.

Keller, N., Jacobson, L.S., Nel, M., Clerq, M., Thompson, P.N., Schoeman, J.P., 2004. Prevalence and risk factors of hypoglycemia in virulent canine babesiosis. Journal of Veterinary Internal Medicine 18, 265-270.

Kettner, F., Reyers, F., Miller, D., 2003. Thrombocytopaenia in canine babesiosis and its clinical usefulness. Journal of the South African Veterinary Association 74, 63-68.

Köster, L.S., Schoor, M., Goddard, A., Thompson, P.N., Matjila, P.T., Kjelgaard-Hansen, M., 2009. C-reactive protein in canine babesiosis caused by Babesia rossi and its association with outcome. Journal of the South African Veterinary Association 80, 87-91.

Kristensen, A.T., Wiinberg, B., Jessen, L.R., Andreasen, E., Jensen, A.L., 2008. Evaluation of human recombinant tissue factor-activated thromboelastography in 49 dogs with neoplasia. Journal of Veterinary Internal Medicine 22, 140-147.

Laforcade, A.M., Freeman, L.M., Shaw, S.P., Brooks, M.B., Rozanski, E.A., Rush, J.E., 2003. Hemostatic changes in dogs with naturally occurring sepsis. Journal of Veterinary Internal Medicine 17, 674-679.

Levi, M., van der Poll, T., ten Cate, H., 2006. Tissue factor in infection and severe inflammation. Seminars in Thrombosis \& Hemostasis 32, 33-39. 
Matjila, P.T., Penzhorn, B.L., Bekker, C.P.J., Nijhof, A.M., Jongejan, F., 2004. Confirmation of occurrence of Babesia canis vogeli in domestic dogs in South Africa. Veterinary parasitology $122,119-125$.

Moore, D.J., Williams, M.C., 1979. Disseminated intravascular coagulation: a complication of Babesia canis infection in the dog. Journal of the South African Veterinary Association 50, 265275.

Murata, H., Shimada, N., Yoshioka, M., 2004. Current research on acute phase proteins in veterinary diagnosis: an overview. Veterinary Journal 168, 28-40.

Nel, M., Lobetti, R.G., Keller, N., Thompson, P.N., 2004. Prognostic value of blood lactate, blood glucose, and haematocrit in canine babesiosis. Journal of Veterinary Internal Medicine 18, 471-476.

Otto, C.M., Rieser, T.M., Brooks, M.B., Russell, M.W., 2000. Evidence of hypercoagulability in dogs with parvoviral enteritis. Journal of the American Veterinary Medical Association 217, 1500-1504.

Rafaj, R.B., Matijatko, V., Kiš, I., Kučer, N., Živičnjak, T., Lemo, N., Žvorc, Z., Brkljačic', M., Mrljak, V., 2009. Alterations in some blood coagulation parameters in naturally occurring cases of canine babesiosis. Acta Veterinaria Hungarica 57, 295-304.

Rafaj, R.B., Mrljak, V., Guelfi, J.F., Marinculic, A., Potocnjak, D., Zvorc, Z., Kucer, N., 2005. Number of thrombocytes and mean platelet volume in canine babesiosis. Revue de Médecine Vétérinaire 156, 95-98.

Ruiz, d.G., Peñalba, B., Goicoa, A., Espada, Y., Fidalgo, L.E., Espino, L., 2007. Clinicopathological findings and coagulation disorders in 45 cases of canine babesiosis in Spain. Veterinary Journal 174, 129-132.

Schetters, T.P.M., Kleuskens, J.A.G.M., Crommert, J., Leeuw, P.W.J., Finizio, A.L., Gorenflot, A., 2009. Systemic inflammatory responses in dogs experimentally infected with Babesia canis; a haematological study. Veterinary parasitology 162, 7-15.

Schoeman, J.P., 2009. Canine babesiosis. Onderstepoort Journal of Veterinary Research 76, 5966.

Schoeman, J.P., Rees, P., Herrtage, M.E., 2007. Endocrine predictors of mortality in canine babesiosis caused by Babesia canis rossi. Veterinary parasitology 148, 75-82.

Schouls, L.M., Van De Pol, I., Rijpkema, S.G., Schot, C.S., 1999. Detection and identification of Ehrlichia, Borrelia burgdorferi sensu lato, and Bartonella species in Dutch Ixodes ricinus ticks. Journal of clinical microbiology 37, 2215-2222. 
Scott-Moncrieff, J., Treadwell, N.G., McCullough, S.M., Brooks, M.B., 2001. Hemostatic abnormalities in dogs with primary immune-mediated hemolytic anemia. Journal of the American Animal Hospital Association 37, 220-227.

Smith, S.A., 2010. Overview of hemostasis. In: Weiss, D.J., Wardrop, K.J. (Eds.), Schalm's Veterinary Hematology. Wiley-Blackwell, Iowa, USA, pp. 635-653.

Stokol, T., 2003. Plasma D-dimer for the diagnosis of thromboembolic disorders in dogs. Veterinary Clinics of North America, Small Animal Practice 33, 1419-1435.

Taylor FB Jr. Toh CH. Hoots WK. Wada H. Levi M. Scientific subcommittee on disseminated intravascular coagulation (DIC) of the International Society on Thrombosis and Haemostasis (ISTH), 2001. Towards definition, clinical and laboratory criteria, and a scoring system for disseminated intravascular coagulation. Thrombosis \& Haemostasis 86, 1327-1330.

Weiss, D.J., Rashid, J., 1998. The sepsis-coagulant axis: a review. Journal of Veterinary Internal Medicine 12, 317-324.

Welzl, C., Leisewitz, A.L., Jacobson, L.S., Vaughan-Scott, T., Myburgh, E., 2001. Systemic inflammatory response syndrome and multiple-organ damage/dysfunction in complicated canine babesiosis. Journal of the South African Veterinary Association 72, 158-162.

Wiinberg, B., Jensen, A.L., Johansson, P.I., Kjelgaard-Hansen, M., Rozanski, E., Tranholm, M., Kristensen, A.T., 2010. Development of a model based scoring system for diagnosis of canine disseminated intravascular coagulation with independent assessment of sensitivity and specificity. Veterinary Journal 185, 292-298.

Wiinberg, B., Jensen, A.L., Johansson, P.I., Rozanski, E., Tranholm, M., Kristensen, A.T., 2008. Thromboelastographic evaluation of hemostatic function in dogs with disseminated intravascular coagulation. Journal of Veterinary Internal Medicine 22, 357-365.

Wiinberg, B., Jensen, A.L., Kjelgaard-Hansen, M., Rojkjaer, R., Johansson, P.I., Gade, L.P., Gram, D.X., Kristensen, A.T., 2007. Study on biological variation of haemostatic parameters in clinically healthy dogs. Veterinary Journal 174, 62-68.

Wiinberg, B., Jensen, A.L., Rozanski, E., Johansson, P.I., Kjelgaard-Hansen, M., Tranholm, M., Kristensen, A.T., 2009. Tissue factor activated thromboelastography correlates to clinical signs of bleeding in dogs. Veterinary Journal 179, 121-129.

Wright, I.G., Goodger, B.V., Buffington, G.D., Clark, I.A., Parrodi, F., Waltisbuhl, D.J., 1989. Immunopathophysiology of babesial infections. Transactions of the Royal Society of Tropical Medicine \& Hygiene 83, 11-13. 